\title{
miRNA in Cancer Pathology
}

\author{
Mouneera Alakeel ${ }^{1} \&$ Zainab Al-Doori ${ }^{2}$ \\ ${ }^{1}$ Clinical Pharmacology, University of Glasgow, Glasgow, Scotland, UK \\ ${ }^{2}$ Library, Saltire Centre, Glasgow Caledonian University, Glasgow, Scotland, UK \\ Correspondence: Zainab Al-Doori, 26Dornoch Way, Cumbernauld, G68 0JA. North Lanarkshire, Scotland, UK. \\ E-mail: zenatulab@gmail.com. Tel: 44-0-786-171-2008.
}

Received: November 1, 2016

Accepted: January 20, 2017 Online Published: February 6, 2017

doi:10.5539/jmbr.v7n1p20

URL: http://dx.doi.org/10.5539/jmbr.v7n1p20

\begin{abstract}
MicroRNA/miRNA refers to types of RNA which are non-coding; they are21 to 25 nucleotides in length. In most cases, at particular nucleotide locations, they relate to one or more mRNAs. Deadenylation, cleavage, and alternative procedures of translation's suppression are the means by which miRNA disturb gene repression. Recent investigations seem to propose that miRNAs are involved in many cell procedures and for this they became perfect targets for usage in healing purposes. Various miRNAs are involved in the development of human vascular diseases, due to their function in modulating vascular cell proliferation, differentiation, migration and apoptosis via their targeted genes. Since vascular diseases are multifactorial and complex, numerous genes may be involved in their progression and regulation. Therefore, miRNAs can also have multiple gene targets, and in some instances, one gene can be modulated by various miRNAs. As of now, more than 800 human microRNAs have been identified using miBase, and work is still being conducted to search for and characterize new miRNAs. With rigorous clinical and fundamental studies, a clear understanding of how miRNAs function, in addition to the ways they can be used as biomarkers and targets for cancer and cardiovascular illnesses, will progress.
\end{abstract}

\section{Aims}

The aim is to provide the basic principles of miRNAs in development of diseases. In this article, the possibility of miRNAs on the clinical application for diagnosis and therapy of diseases will be reviewed and discussed.

Here, a systematic search of the literature to review and present the currently available evidence on the possible roles of microRNAs was conducted.

\subsection{Background}

MicroRNAs constitute a large family of approximately 21-nucleotide-long, noncoding RNAs. They emerged more than 20 years ago as key post transcriptional regulators of gene expression. MicroRNAs exist remarkably in body fluids and are steadily protected from endogenous RNase activity (Eisenberg et al., 2015). Evidence has been offered that microRNA expression when dysregulated then it may play an essential role in development and spreading of diverse cancers including ovarian cancer (Virant-Klun et al., 2016). Thus, miRNAs play fundamental roles in carcinogenesis, but the global miRNA expression profile in gastric stromal cancer tissues continued uncertain.

Each miRNA can control a numerous target genes and play vital roles in many biological procedures including organogenesis, haematopoiesis, cell development, proliferation, and invasion ( $\mathrm{Yu} \& \mathrm{Li}, 2015)$; Micro-RNA regulates gene expression by intermingling with messenger RNA (Tuna et al., 2016).

\section{Recent Studies and Results:}

MicroRNAs are found to perform an essential role in the regulation of reproductive functions, such as oocyte development, folliculogenesis, corpus luteum function, implantation, and early embryogenesis (Eisenberg et al., 2015). In this paper, the most recent information on microRNAs, precisely correlating to human gonadal cells was discussed. The dysregulation of miRNAs plays a role in the development of the cancer as well as viral interference. It has been shown that many miRNAs are deregulated by HBV infection and participate in viral reproduction and pathogenesis (Naito et al., 2015). Thus, it is proposed that the exact mechanism between miRNA and HBV natural science will result in the expansion of the new diagnostics and therapies. Cell-free miRNAs have been suggested as biomarkers of disease, including diagnosis, prognosis, and watching treatment responses (Leuenberger \& Saugy, 2015). These circulating miRNAs are steadily stable in many body fluids such as plasma and serum thus 
the profiles of flowing miRNAs have been discovered in the area of anti-doping. Integrating longitudinal dimensions of circulating miRNAs into the Athlete Biological Passport is suggested as an effective approach for their employment as novel biomarkers (Leuenberger \& Saugy, 2015); in their paper, probable experiments related to the conversion of measurements of circulating miRNAs from research situations to applied anti-doping presentations were offered.

Katsura et al. (2016) established that microRNAs which are constitutively active, and so are the inducible microRNAs, they function as phenotypic transformers interrelated with transcriptome dynamics during EndMT, the endothelial-mesenchymal transition (EndMT) elaborate in / and pathogenesis. From their work, Katsura et al. (2016) found that miR-31 functions as a molecular hub to integrate TGF- $\beta$ and TNF- $\alpha$ signalling to enhance EndMT.

In early 2016, Virant-Klun et al. (2016) abridged the current understanding of microRNAs correlated to woman's fertility and cancer: from primal germ cells and ovarian function, germinal stem cells, oocytes, and embryos to embryonic stem cells.

Cell-derived vesicles or exosomes can carry microRNAs and also proteins between different cells and are involved in cell-to-cell communication. Virant-Klun et al. (2016) confirmed in their conclusion that microRNAs, together with exosomes, have a great possibility for their use in prognosis, and therapy. Furthermore, they can be used as biomarkers of different diseases as well as infertility. RNase L protects from pathogens and controls cell growth and differentiation by disrupting largely unidentified mammalian RNA targets. Rath et al. (2015) revealed that RNase L-dependent decay selectively disturbs transcripts controlled by microRNA (miR)-17/miR-29/miR-200 and other miRs that function as suppressors of mammalian cell adhesion and multiplication. RNase L acts as a suppressor of proliferation and adhesion in mammalian cells. Rath et al. (2015) concluded that RNase L-dependent decay starts an anti-proliferative state via disruption of the miR-controlled transcriptome.

Recently, a novel web means was presented, the CircInteractome (circRNA interactome), for mapping RBP- and miRNA-binding sites on human circRNAs (Dudekula et al., 2016). Circular RNA, CircRNAs have been demonstrated to perform as sponges for miRNAs and to possibly sponge RNA-binding proteins (RBPs) and are thus expected to act as vigorous post transcriptional regulators of gene expression. The CircInteractome provided bio-informatic analyses of binding sites on circRNAs. It allows the user's ability to:

1) Identify potential circRNAs which functions as RBP sponges

2) Design junction-spanning primers for specific detection of circRNAs of concern

3) Design siRNAs for circRNA silencing

4) Identify potential internal ribosomal entry sites (IRES).

Genetic variants in miRNAs or miRNA binding sites on their target genes could disturb miRNA function and contribute to disease risk (Ghanbari et al., 2016). In this project, Ghanbari et al. (2016) examined the relationship of miRNA-related genetic variants with Parkinson Disease (PD) using data from the largest GWAS on PD. It has been shown that the variant's mutant alleles alter the secondary structure and reduce the expression level of their correlated miRNAs. Among 48,844 miRNA binding site-variants, 32 variants (within 13 genes) have been found that are connected with PD. Evidence supporting the influence of the identified miRNA binding site-variants on miRNA-mediated regulation of their host genes has been provided (Ghanbari et al., 2016).

Petrozza et al. (2015) aimed to identify specific miRNAs that are decontrolled in tumour vs regular kidney tissues and that could influence on the biology of clear cell renal cell carcinoma (ccRCC). In their study, Petrozza et al. (2015) described the identification of specific oncogenic miRNAs that are changed in ccRCC tissues and suggested that they might be useful biomarkers in ccRCC management.

It has been shown that miR-34a directly binds to the 3' untranslated region of RAD51 and regulates homologous recombination, preventing double-strand-break repair in NSCLC cells (Cortez et al., 2015). MiR-34a, a significant tumour-suppressing microRNA, and is downregulated in numerous types of cancer. Furthermore, Cortez et al. (2015) demonstrated the therapeutic prospective of miR-34a transfer in combination with radiotherapy in mouse models of lung cancer. In conclusion, the authors recommended that administration of miR-34a in combination with radiotherapy may represent a new strategy for treating NSCLC (Cortez et al., 2015). Xiao et al. (2015) aimed to inspect the miRNA expression outline in gastric stromal tumour tissues and to explore the role of dysregulated miRNAs by carrying out gene ontology (GO) and pathway improvement analysis; as a result of their research Xiao et al. (2015) showed that there were 12 differently expressed microRNAs in gastric stromal tumour tissues, among which 10 miRNAs were down-controlled, and 2 were up-controlled $(\mathrm{P}<0.05)$. Gene ontology (GO) analysis revealed that the target genes of miR-3178 were involved in 5 GO terms and those of miR-193a-5p in 7 GO terms in level 2. It was concluded that gastric stromal tumour displays a unique miRNA signature (Xiao et al., 2015). 
This precise expression may develop a new diagnostic and prognostic biomarker for gastric stromal tumour. Furthermore, it is concluded that miR-3178 and miR-193a-5p function as suppressive microRNAs, and they may also become diagnosis and treatment targets for gastric stromal tumour (Xiao et al., 2015).

Overexpression of miR-155 is pathogenic in B-cell malignancy but was also described in many solid tumoursespecially, in breast cancer, where its role is uncertain and often inconsistent (Goldgraben et al., 2016). In their research, the growth of six breast cancer cell lines transfected with numerous miRNA mimics was analysed. Goldgraben et al. (2016) found that MCF-7 cell growth to be inhibited by miR-155 and miR-145 mimics, both 23$\mathrm{nt}$ long, but not by a number of shorter mimics, including the negative control. Apoptosis is brought, accompanied with interferon B after stimulation of the double stranded RNA sensor pathway. A panel of breast cancer cell lines revealed an extensive series of sensitivities to 23-mer mimics, broadly consistent with the sensitivity of the cell lines to Poly (I:C). Two bases for nonspecific effects by miRNA mimics: duplex length and the artificial passenger strand were established (Goldgraben et al., 2016). Moreover, the risk of a universal 21-mer negative control and the significance of using matched seed mutants for consistent understanding of phenotypes, were stressed (Goldgraben et al., 2016).

$\mathrm{Yu}$ and $\mathrm{Li}$ (2015) presented cumulative evidences presenting that miRNAs have a vital role in adrenocortical carcinomas (ACCs). Abnormal miRNA expression may contribute to ACC carcinogenesis, and it can be a tumoursuppressive or oncogenic miRNAs. Yu and Li (2015) in their review abridged the influence of miRNAs to the introduction and progress of ACCs.

MicroRNAs (miRNAs) from the miR-371-373 and miR-302/367 clusters are overexpressed in all malignant germcell tumours (GCTs) (Murray et al., 2016). Some of these miRNAs demonstrate an elevation in serum levels at diagnosis. A forceful technical pipeline has been developed to quantify these miRNAs in serum and cerebrospinal fluid (CSF). The pipeline was used in samples from a cohort of entirely paediatric patients with gonadal and extra gonadal malignant GCTs, related with suitable tumour and non-tumour control groups (Murray et al., 2016). A four-serum miRNA panel (miR-371a-3p, miR-372-3p, miR-373-3p and miR-367-3p): (i) showed high sensitivity/specificity for diagnosing paediatric extracranial malignant GCT; (ii) allowed early recognition of decline of a testicular mixed malignant GCT; and (iii) Identified intracranial malignant GCT distinctly from intracranial non-GCT tumours using CSF and serum samples. It is concluded that the pipeline theoretically potentials to progress clinical controlling of both paediatric and adult malignant GCTs (Murray et al., 2016).

Kirby et al (2016) suggested that use of bio-informatics incorporation of mRNA and microRNA arrays produces confirmable mRNA-microRNA pairs that are accompanying insulin resistance, and can be confirmed in vitro. In their study, an integrative mRNA-mi-RNA microarray method for the purpose of identifying putative molecular interactions that control the transcriptome in subcutaneous adipose tissue of insulin sensitive (IS) and insulin resistant (IR) people, has been used (Kirby et al., 2016). Seventeen mi-RNAs have been revealed that they are differentially expressed in insulin resistant (IR) versus insulin sensitive (IS). $94 \%$ of them were down-regulated in IR versus IS, including miR-26b, miR-30b, and miR-145. Furthermore, the genes that were expected targets of miR-26b, miR-30b and miR-145, were recognised and were up-regulated in IR people (Kirby et al., 2016).

Micro-RNA regulates gene expression by intermingling mainly with m- RNA. Recently, Tuna et al. (2016) reviewed the disruption of miRNAs and their key regulatory machineries by genetic alterations, with emphasis on mutations and epigenetic changes in cancer and other diseases. Changes in these regulatory genes may result in disease, such as cancer. Disruption of these genes can occur through epigenetic and genetic alterations, resulting in abnormal expression of miRNAs and consequently of their target genes (Tuna et al., 2016).

Rabiee - Ghahfarrokhi et al. (2015) have recently utilized an efficient genetic algorithm-based decision tree to forecast microRNA target genes as their first step to disentangle the role of a specific miRNA is the recognition of its direct targets. A genetic algorithm in association with C4.5 decision tree for expectation of miRNA targets has been used in this project. Their proposed method has been applied to an authorized human datasets (Rabiee Ghahfarrokhi et al. (2015). The Precision of classification of $93.9 \%$ has been accomplished, which could be due to the best rules' selection.

Interestingly, the role of miRNAs in controlling $\mathrm{K}+$ channels of epithelial tissues is only developing now and Pilmore and Hamilton (2015) were concentrating on the recent new findings into miRs in the regulation of $\mathrm{K}+$ channels of epithelia.

Recently, the supposed role of miR-200b in the development of glioma has been investigated (Li et al., 2016). It has been revealed that the miR-200b levels were often reduced in primary glioma tissues $(n=88)$ and cell lines, when related to normal brain tissues $(\mathrm{n}=25)$. Reduced miR-200b level was strongly linked with the malignant development of glioma (Li et al., 2016). ZEB2 has been recognized as a direct target of miR-200b, and the protein 
expression of ZEB2 was evidently reduced after overexpression of miR-200b in U251 and U87 cells. Overexpression of miR-200b, in vivo, considerably repressed tumorigenesis and tumour growth of glioma cells, as well as successfully protected nude mice from tumour-induced death. Li et al. (2016) advised that miR-200b has suppressive properties on the propagation, migration, invasion and EMT of glioma cells, partially at least, via targeting ZEB2. Therefore, it is concluded that miR-200b role as a new tumour suppressor in glioma, and thus may become a favourable therapeutic candidate for glioma ( $\mathrm{Li}$ et al., 2016).

Dysregulated microRNAs (miRs) control the pathogenesis of Non-alcoholic fatty liver disease (NAFLD). In Xiao et al. (2016) study, normal or high-fat diet (HF) mice were either exposed to a 16-week in a row program or retained inactive. Exercise weakened liver steatosis in HF mice. It is shown that miR-212 was overexpressed in HF liver, while reduced by exercise (Xiao et al., 2016). Furthermore, the role of miR-212 in lipogenesis using HepG2 cells with/without long-chain fatty acid treatment ( \pm FFA) was examined. FFA augmented miR-212 in HepG2 cells and miR-212 stimulated lipogenesis in HepG2 cells ( \pm FFA). Also, miR-212 negatively controls fibroblast growth factor (FGF)-21 at protein level in HepG2 cells. However, FFA downregulated FGF-21 both at mRNA and protein levels in HepG2 cells. Moreover, siRNA-FGF-21 eradicated the lipogenesis-reducing effect of miR-212 inhibitor in HepG2 cells ( \pm FFA), confirming FGF-21 as a target gene of miR-212 (Xiao et al., 2016).

Another mi-RNA, namely miR-512-5p has been discovered to encourage apoptosis and to inhibit glycolysis by targeting p21 protein in non-small cell lung cancer cells (Chu et al., 2016).In their study, Chu et al. (2016) demonstrated that the expression of miR-512-5p is downregulated in non-small cell lung cancer (NSCLC) patient tumour examples. Overexpression of miR-512-5p induced apoptosis of NSCLC cell lines A549 and H1299, and miR-512-5p inhibitor overturned this influence in H1299 cells. MiR-512-5p inhibited glycolysis and migration in NSCLC cells, but does not influence cell proliferation (Chu et al., 2016). P21 has been identified as the target gene of miR-512-5p thus the overexpression of miR-512-5p resulted in the decrease of p21 protein and mRNA level. $\mathrm{Chu}$ et al. (2016) concluded that their investigation revealed miR-512-5p capability to target $\mathrm{p} 21$ to induce apoptosis and inhibit glycolysis in A549 and H1299 cell lines.

More recent studies aim for the identification of the role of microRNA including the role of a new tumour suppressor microRNA (miR) and searching for its physiological role and mechanism in renal cell carcinoma (RCC) (Zou et al., 2016). Reverse transcription-quantitative polymerase chain reaction analysis has been used to measure miRNA (miR)-362-3p expression levels in 47 pairs of RCC and neighbouring normal tissue samples. Research on the role of miRNA (miR)-362-3p in the regulation of cell proliferation, migration, invasion, apoptosis and cell cycle was carried out (Zou et al., 2016). Luciferase reporter assays and western blot analyses were carried out to discover the target gene for miRNA (miR)-362-3p. It has been shown that the expression levels of miR-362-3p were downregulated in the RCC tissue samples. The upregulation of miR-362-3p using a synthesized mimic suppressed the propagation, migration and invasion of the renal cancer cells, and induced cell apoptosis and G1 phase halt in addition to a reduced expression levels of nemo-like kinase (Zou et al., 2016); for this it is concluded that miR-362-3p role is as a tumour suppressor in RCC, and thus may function as a probable molecular target in the management of RCC.

The regulatory role of microRNAs (miRNAs) in the control of tumour metastases has been thoroughly designated in several types of cancer as described earlier in this review but the significance in bone metastasis of prostate cancer (PCa) stayed largely unidentified until Fu et al. (2015) investigated this. In their study, the differentially expressed miRNAs were identified between the primary $\mathrm{PCa}$ and bone metastatic $\mathrm{PCa}$ examples by associating their expression profiling using miRNA microarray. As a result of their research, 4 miRNAs (miR-335, -543, -196 and -19 a) were distinguished to be significantly downregulated in bone metastasis in relation with primary PCa. By the application of reverse transcription-quantitative polymerase chain reaction analysis, the down regulation of miR-335 and miR-543 was established in a total of 20 paired primary PCa and bone metastasis samples (Fu et al., 2015). Also, by inspecting the expression pattern of endothelial nitric oxide synthase, eNOS, in primary PCa and skeletal metastatic samples, the mRNA and protein expression levels of eNOS were noticeably upregulated in the metastatic samples. In addition, Fu et al. (2015) showed that exogenous overexpression of miR-335 and -543 considerably downregulated the level of expression of eNOS, and significantly negotiated the capability of migration and invasion in vitro. It is suggested that miR-335 and -543 are concomitant with bone metastasis of $\mathrm{PCa}$ and designated that they may have vital roles in the bone metastasis, which may also be clinically used as novel biomarkers in discerning the different stages of human $\mathrm{PCa}$ and predicting bone metastasis.

Lee et al. (2015) aimed to disclose the regulatory miRNA-target gene relations that arise differentially in inflamed small- compared with large-IECs, intestinal-epithelial cells. Whereas inflammatory bowel diseases, IBD, considerably increased the expression of only a few miRNA candidates in small-IECs, numerous miRNAs were upregulated in inflamed large-IECs. This is an evidence of the sensitivity of large intestine to colitis in comparison 
to small intestine. It is proposed that miRNAs such as miR-1224, miR-3473a, and miR-5128 symbolize biomarkers that appear in large-IECs upon IBD progress and co-operatively suppress the expression of key anti-inflammatory factors (Lee et al., 2015).

MiR-146a has been recognized as a new anti-inflammatory noncoding RNA modulator of diabetic nephropathy, DN (Bhatt et al., 2015). It has been shown that Diabetes-induced upregulation of pro-inflammatory and pro-fibrotic genes were considerably grander in the kidneys of miR-146a-/- than in the kidneys of wild-type mice (Bhatt et al., 2015). MiR-146a lack during diabetes resulted in increased expression of M1 stimulation markers and suppression of M2 markers in macrophages. These studies propose that in early DN, miR-146a upregulation applies a protective effect by downregulating target inflammation-related genes, leading to suppression of pro-inflammatory and inflamma-some gene activation (Bhatt et al., 2015).

MicroRNAs (miRs) normally act as regulators of gene expression through binding to target mRNAs. Wang et al. (2016) have recently emphasized the pathogenesis of chronic myeloid leukaemia (CML), functional significance of miRs and lncRNAs (long non-coding RNAs) in regulation of CML progress and complicated mechanisms fundamental to their action.

Kaudewitz et al. (2016) believed that it is uncertain whether plasma miRNA levels are related to platelet function. For this they aimed to link small RNAs to platelet reactivity. Using quantitative real-time polymerase chain reactions, 92 miRNAs were assessed in acute coronary syndrome (ACS) patients on different anti-platelet treatments. Key platelet-related miRNAs and YRNAs were correlated with platelet function. MiR-223 and miR126, other copious platelet miRNAs and YRNAs exhibited significant positive correlations with the vasodilatorstimulated phosphoprotein phosphorylation assay. Kaudewitz et al. (2016) also showed that YRNAs, miR-126 and miR-223 were also amongst the small RNAs showing the utmost reliance on platelets. In addition, a single nucleotide polymorphism that enables processing of pri-miR-126 to mature miR-126 accounted for a rise in circulating platelet stimulation markers. Finally, inhibition of miR-126 in mice reduced platelet accumulation and it directly and indirectly affects ADAM9 and P2Y12 receptor expression. It is concluded that levels of plateletrelated plasma miRNAs and YRNAs correlate with platelet function tests in ACS patients and platelet stimulation markers in the general population and that changes in miR-126 affect platelet reactivity (Kaudewitz et al., 2016). An experimental-validated map of RAS-MAPK oncomiRs and tumour suppressor miRNAs from transmembrane receptor to downstream ERK proteins has been recently provided (Masliah-Planchon et al., 2016).According to which, MiRNAs could be further regarded as probable genetic biomarkers for diagnosis, prognosis, or therapeutic purpose. Mounting proof had suggested the importance of miRNAs targeting the RAS-MAPK in oncogenesis. MiRNAs changes in human cancers may act as a rheostat of the oncogenic RAS signal that is frequently augmented as cancers' development (Masliah-Planchon et al., 2016).

Lee et al. (2016) aimed to detect the specific miRNAs involved in regulation of EIF4EBP1 expression in ovarian cancer and to describe their natural role. They showed that miR-125a and miR-125b were downregulated in ovarian cancer tissue and cell lines relative to healthy controls. The inverse relationship between miR-125a and miR-125b was supported by co-transfection of a luciferase reporter plasmid. They both, namely miR125a and miR125b instigated apoptosis and reduced cell viability and migration in an apparently EIF4EBP1-directed means (Lee et al., 2016). It is concluded that miR-125a and miR-125b are significant post transcriptional regulators of EIF4EBP1 expression, providing reasoning for novel therapeutic methods to suppress tumour invasion and migration using miR-125a, miR-125b, or their mimics for the treatment of ovarian cancer (Lee et al., 2016).

Melo et al. (2015) aimed to assess the effect of exercise training on cardiac microRNA-1 and -214 expressions after myocardial infarction. Melo's group showed that MicroRNA-1 and -214 expressions were, respectively, decreased and increased in the sedentary infarcted rats ,S-INF, compared to the sedentary sham, S-SHAM, rats while exercise working out regularized the expression of these microRNAs. The microRNA targets sodium/calcium exchanger 1, NCX and sarcoplasmic reticulum calcium ATPase-2a, Serca-2a protein expressions were, respectively, lessened (55\%) and increased (34\%) in the training infarcted, T-INF group compared to the $\mathrm{S}-\mathrm{INF}$ group. It is worth noting that these groups are involved in cardiac function ruling. This group of researchers concluded that exercise working out re-establishes microRNA-1 and -214 expression levels and inhibits alteration in both NCX and Serca-2a protein and gene expressions. Finally, Melo et al. (2015) proposed a molecular mechanism to re-establish ventricular function post exercise training in myocardial infarction rats.

Since microRNAs (miRNAs) play essential role during cancer metastases, determination of the involved miRNAs in ruling of the metastases of bladder cancer (BC) may offer new therapeutic goals for BC treatment. This has encouraged Sun et al. (2015) to carry out a research on the role of miR-138 in regulation of BC cell invasion and metastases. The levels of miR-138 and ZEB2, an essential factor that regulates cancer cell invasion, were examined 
in the BC samples from the patients. The binding of miR-138 to the 3'-UTR of ZEB2 mRNA was established by bioinformatics analysis, and the natural influence of this binding through promoter luciferase reporter assay was confirmed (Sun et al., 2015). It is found that the levels of miR-138 were suggestively reduced and the levels of ZEB2 were considerably increased in BC samples. So, miR-138 and ZEB2 were inversely correlated in BC samples. MiR-138 targeted the 3'-UTR of ZEB2 mRNA to prevent its translation, as bioinformatics examination shown. Finally, Sun et al. (2015) showed that, miR-138 overexpression prevented ZEB2-mediated cell invasion and metastases, while miR-138 reduction increased ZEB2-mediated cell invasion and metastases in BC cells.

In conclusion, suppression of miR-138 in BC cells may encourage ZEB2-mediated cancer invasion and metastases. Thus, miR-138 seems to be an intriguing therapeutic target to prevent metastases of bladder cancer (Sun et al., 2016).

MicroRNAs (miRNAs) have a significant role in epithelial-to-mesenchymal transition (EMT) and hyperglycaemia induces destruction of renal tubular epithelial cells, which may result in EMT in diabetic nephropathy (Liu et al., 2016). In their study, the level of miR-23b was greatly reduced in high glucose (HG)-induced human kidney proximal tubular epithelial cells (HK2) and in kidney tissues of $\mathrm{db} / \mathrm{db}$ mice. Also, miR-23b repressed EMT in diabetic nephropathy by affecting high mobility group A2 (HMGA2), thereby suppressing PI3K-AKT signalling pathway stimulation. Liu et al. (2016) also showed that HMGA2 overexpression or activation of the PI3K-AKT signalling pathway with $\mathrm{BpV}$ prohibited the effects of miR-23b on HG-mediated EMT. It is established that overexpression of miR-23b relieved EMT, reduced the expression levels of EMT-related genes, enhanced renal morphology, glycogen build-up, fibrotic reactions and enhanced renal functions in $\mathrm{db} / \mathrm{db}$ mice (Liu et al., 2016). Thus it was found in this study that miR-23b acts as a suppressor of EMT in diabetic nephropathy through repressing PI3K-AKT signalling pathway stimulation by targeting HMGA2, which maybe a probable therapeutic target for diabetes-induced renal dysfunction.

Chronic hepatitis B (CHB), chronic hepatitis C (CHC), and accompanying hepatocellular carcinoma (HCC) are characterized by cholesterol disparity and dyslipidaemia as distinguished by Selitsky et al. (2015). The controlling mechanisms fundamental to the dysregulation of lipid pathways in these disease conditions are partly understood. For this, a combination of genomic, molecular, and biochemical approaches to recognize significant miRNAs that drive the lipid phenotypes of chronic viral hepatitis and HCC has been accomplished (Selitsky et al., 2015). Integrative analysis of microRNA (miRNA) has been done as well as gene expression in non-malignant and matched cancer tissue samples from human subjects with CHB or CHC and HCC. In addition, a continuation of efficient study of definite miRNAs in a cell-based system has been carried out.

Firstly, it is found that, pathways influencing cholesterol homeostasis were amongst the most importantly overrepresented among genes dysregulated in chronic viral hepatitis and specifically in tumour tissue. Secondly, specific miRNA signatures that involved miRNAs not until that time associated with chronic viral hepatitis, such as miR-1307 in CHC, were recognized for each disease state namely: $\mathrm{CHB}, \mathrm{CHC}, \mathrm{CHB}$-accompanying $\mathrm{HCC}$, and CHC-accompanying HCC. Thirdly, candidate master miRNA controllers of pathways regulating cholesterol homeostasis in chronic viral hepatitis and HCC has been identified, including miR-21, miR-27, and miR-33. Finally, it has been confirmed that miR-21 and miR-27 considerably repress cholesterol synthesis and that miR27 does so partly through regulation of the gene coding for the rate-limiting enzyme 3-hydroxy-3-methyl-glutarylcoenzyme A (HMG-CoA) reductase (HMGCR) (Selitsky et al., 2015). That is evidence that micro-RNAs contribute to the progress of new and more-effective miRNA-based therapeutic approaches.

Recently, it has been found that miR-485 of the host was formed in response to viral infection and targeted RIG-I mRNA for degradation where RIG-I is retinoic acid-inducible gene I. this resulted in suppression of the antiviral response and boosted viral replication (Ingle et al., 2015). In mammalian cells, mir-485 inhibition of its expression has significantly reduced the replication of Newcastle disease virus (NDV) and the H5N1 strain of influenza virus. Ingle et al. (2015) also showed that miR-485 demonstrated bi-specificity, targeting RIG-I in cells with a low abundance of $\mathrm{H} 5 \mathrm{~N} 1$ virus and targeting PB1 (which encodes an RNA polymerase necessary for viral replication) in cells with augmented amounts of the $\mathrm{H} 5 \mathrm{~N} 1$ virus.

Fang et al. (2015) questioned the variation of micro RNA expression by substance P (SP) which is a neuropeptide associate of the tachykinin family that has a serious role in colitis. Real time polymerase chain reaction, RT-PCR, was useful for the confirmation of targets of SP-regulated microRNAs. Roles of miRNAs were confirmed in NCM460-NK-1R cells and the TNBS and DSS models of colitis. A novel NK-1R-miR-221-5p-IL-6R network that protects from colitis is shown; thus it is concluded that the use of miR-221-5p mimics may be an encouraging approach for colitis cure (Fang et al., 2015). SP stimulated differential expression of 29 microRNAs, including miR-221-5p, the highest up regulated miR (by 12.6-fold) upon SP activation. Interleukin 6 receptor (IL-6R) mRNA has been recognized as a direct targets of miR-221-5p in NCM460 cell. NCM460-NK-1R cells increased IL-6R 
mRNA expression when exposure to SP whereas overexpression of miR-221-5p decreased IL-6R expression (Fang et al., 2015).Furthermore, it is found that MiR-221-5p expression was elevated in both TNBS- and DSS-induced colitis. In situ hybridization in TNBS-and DSS-exposed colons revealed augmented miR-221-5p expression primarily in colonocytes (Fang et al., 2015).

Exosome is a kind of biological membrane structure at nanometre level. It is secreted by several cells in the body and broadly spread in utmost body fluids, like saliva, milk, and blood (Tang et al., 2015). Biological active substances, including mRNAs, microRNAs, cytokines, and transcription factor, have been well-known in the exosomes. With several biological roles, exosome-derived miRNAs can not only be functioned as biomarkers to diagnose cancer, neurological diseases and mental disorders, but also have possible role as treatment targets (Tang et al., 2015).

MicroRNAs (miRNAs) are alike in their significance to transcription factors and serious to convene accuracy and strength in gene expression programs and subsequently, have appeared as regulators of the immune response (Gonzalez-Rascón \& Mata-Haro, 2015). One suggested mechanism of probiotic effect on immune responses includes the receptor-mediated interaction of dendritic cells with constituents of the cellular membrane and/or secreted peptides of probiotics finishing with the production of cytokines. Proteins as TNF receptor-associated factor 6 (TRAF6), Interleukin-1 receptor-associated kinase 4 (IRAK4) and Interleukin-1 receptor-associated kinase 3 (IRAK3 or IRAKM) contribute in a significant way in the nuclear factor kappa B (NF-kB) signalling pathway, which is the key cascade stimulated in response to probiotics. These proteins are controlled by miRNAs and 22contribution of miRNAs controlling the TLR2/NF- $\mathrm{kB}$ pathway in the native immune response facilitated by probiotics.

\subsection{Points of Dispute or Unanswered questions:}

It has been recently found that age and gender could affect the pattern of circulating miRNAs (Keller and Meese, 2016). Also, that on the cellular level, the miRNA arrangement varies between plasma and serum preparations. On the molecular level, one has to distinguish between extracellular miRNAs that are captured in micro-vesicles or destined to proteins or high-density lipoproteins (Keller \& Meese, 2016). It is concluded that functional signal for a true miRNA should be gained by cloning the predecessor miRNA and by consequent uncovering of the treated mature form in host cells.

\subsection{Potential Research/ Future}

Zhang et al (2015) utilised the main genes and miRNAs accompanying laryngeal cancer (LC) to make three regulatory networks (differentially expressed, LC-related and global). The network on the development of laryngeal cancer required 10 differentially expressed miRNAs and 55 differentially expressed genes. The pathogenesis of laryngeal cancer has been explained which should be favourable for future research (Zhang et al., 2015).

Finally, Li et al. (2016) researched the lncRNA expression signature in Xuanwei lung cancer (XWLC) by applying microarray assay and bioinformatics analysis. Also, quantitative reverse transcription-polymerase chain reaction (qRT-PCR) was applied to confirm the expression levels of 6 candidate lncRNAs in 33 additional paired XWLC samples. About 37,000 lncRNAs and 34,000 mRNAs were considered in their micro-array. 1,484 lncRNAs and 1,997 mRNAs were differentially expressed in XWLC and neighbouring normal samples (fold change $\geq 2.0$ ) (Li et al., 2016). The qRT-PCR results were consistent with the microarray data as six candidate lncRNAs were differentially expressed in XWLC and adjacent normal tissues. Further examination of the differentially expressed lncRNAs may work as new biomarkers for identification of XWLC or novel therapeutic targets (Li et al., 2016).

\section{References}

Bhatt, K., Lanting, L. L., Jia, Y., Yadav, S., Reddy, M. A., Magilnick, N., ... \& Natarajan, R. (2015). Antiinflammatory role of microRNA-146a in the pathogenesis of diabetic nephropathy. Journal of the American Society of Nephrology, ASN-2015010111. http://dx.doi.org/10.1681/ASN.2015010111

Chu, K., Gao, G., Yang, X., Ren, S., Li, Y., Wu, H., ... \& Zhou, C. (2016). miR-512-5p induces apoptosis and inhibits glycolysis by targeting p21 in non-small cell lung cancer cells. International journal of oncology, 48(2), 577-586. http://dx.doi.org/10.3892/ijo.2015.3279

Cortez, M. A., Valdecanas, D., Niknam, S., Peltier, H. J., Diao, L., Giri, U., ... \& Heymach, J. V. (2015). In vivo delivery of miR-34a sensitizes lung tumors to radiation through RAD51 regulation. Molecular TherapyNucleic Acids, 4, e270. 
Dudekula, D. B., Panda, A. C., Grammatikakis, I., De, S., Abdelmohsen, K., \& Gorospe, M. (2016). CircInteractome: a web tool for exploring circular RNAs and their interacting proteins and microRNAs. RNA biology, 13(1), 34-42.

Eisenberg, I., Kotaja, N., Goldman-Wohl, D., \& Imbar, T. (2015). microRNA in Human Reproduction. Adv Exp Med Biol., 888, 353-387.

Fang, K., Sideri, A., Law, I. K. M., Bakirtzi, K., Koon, H. W., Oikonomopoulos, A., ... \& Pothoulakis, C. (2015) Identification of a novel substance P (SP)-neurokinin-1 receptor (NK-1R) microRNA-221-5p inflammatory network in human colonic epithelial cells. Cell Mol Gastroenterol Hepatol., 1(5), 503-515.

Fu, Q., Liu, X., Liu, Y., Yang, J., Lv, G., \& Dong, S. (2015). MicroRNA-335 and-543 suppress bone metastasis in prostate cancer via targeting endothelial nitric oxide synthase. International journal of molecular medicine, 36(5), 1417-1425. http://dx.doi.org/10.3892/ijmm.2015.2355.

Ghanbari, M., Darweesh, S. K., Looper, H. W., Luijn, M. M., Hofman, A., Ikram, M. A., ... \& Dehghan, A. (2016). Genetic variants in microRNAs and their binding sites are associated with the risk of Parkinson disease. Human mutation. http://dx.doi.org/10.1002/humu.22943

Goldgraben, M. A., Russell, R., Rueda, O. M., Caldas, C., \& Git, A. (2016). Double-stranded microRNA mimics can induce length-and passenger strand-dependent effects in a cell type-specific manner. $R N A, 22(2), 193-$ 203. http://dx.doi.org/10.1261/rna.054072.115

González-Rascón, A., \& Mata-Haro, V. (2015). MicroRNAs: Regulators of TLR2-Mediated Probiotic Immune Responses. MicroRNA, 4(3), 168-174.

Ingle, H., Kumar, S., Raut, A. A., Mishra, A., Kulkarni, D. D., Kameyama, T., ... \& Kumar, H. (2015). The microRNA miR-485 targets host and influenza virus transcripts to regulate antiviral immunity and restrict viral replication. Sci Signal, 8, ra126. http://dx.doi.org/10.1126/scisignal.aab3183

Katsura, A., Suzuki, H. I., Ueno, T., Mihira, H., Yamazaki, T., Yasuda, T., ... \& Miyazono, K. (2016). MicroRNA31 is a positive modulator of endothelial-mesenchymal transition and associated secretory phenotype induced by TGF- $\beta$. Genes to Cells, 21(1), 99-116. http://dx.doi.org/10.1111/gtc.12323..

Kaudewitz, D., Skroblin, P., Bender, L. H., Barwari, T., Willeit, P., Pechlaner, R., ... \& Chan, M. V. (2016). Association of microRNAs and YRNAs with platelet function. Circulation research, CIRCRESAHA-114. http://dx.doi.org/10.1161/CIRCRESAHA.114.305663

Keller, A., \& Meese, E. (2016). Can circulating miRNAs live up to the promise of being minimal invasive biomarkers in clinical settings?. Wiley Interdisciplinary Reviews: RNA, 7(2), 148-156. http://dx.doi.org/10. 1002/wrna. 1320

Kirby, T. J., Walton, R. G., Finlin, B., Zhu, B., Unal, R., Rasouli, N., ... \& Kern, P. A. (2016). Integrative mRNAmicroRNA analyses reveal novel interactions related to insulin sensitivity in human adipose tissue. Physiological genomics, 48(2), 145-153. http://dx.doi.org/10.1152/physiolgenomics.00071.2015

Lee, J., Park, E. J., Yuki, Y., Ahmad, S., Mizuguchi, K., Ishii, K. J., ... \& Kiyono, H. (2015). Profiles of microRNA networks in intestinal epithelial cells in a mouse model of colitis. Scientific reports, 5, 18174-18174. http://dx.doi.org/10.1038/srep18174

Lee, M., Kim, E. J., \& Jeon, M. J. (2016. MicroRNAs 125a and 125b inhibit ovarian cancer cells through posttranscriptional inactivation of EIF4EBP1. Oncotarget, 7(8), 8726-8742. http://dx.doi.org/10.18632/ oncotarget. 6474

Leuenberger, N., \& Saugy, M. (2015). Circulating microRNAs: The Future of Biomarkers in Anti-doping Field. In microRNA: Medical Evidence (pp. 401-408). Springer International Publishing.

Li, J., Yuan, J., Yuan, X., Zhao, J., Zhang, Z., Weng, L., \& Liu, J. (2016). MicroRNA-200b inhibits the growth and metastasis of glioma cells via targeting ZEB2. International journal of oncology, 48(2), 541-550. http://dx.doi.org/10.3892/ijo.2015.3267

Li, Q., Wu, C., Song, G., Zhang, H., Shan, B., Duan, Y., \& Wang, Y. (2016). Genome-Wide Analysis of Long Noncoding RNA Expression Profiles in Human Xuanwei Lung Cancer. Clinical laboratory, 61(10), 15151523.

Liu, H., Wang, X., Liu, S., Li, H., Yuan, X., Feng, B., ... \& Li, H. (2016). Effects and mechanism of miR-23b on glucose-mediated epithelial-to-mesenchymal transition in diabetic nephropathy. The international journal of biochemistry \& cell biology, 70, 149-160. http://dx.doi.org/10.1016/j.biocel.2015.11.016 
Masliah-Planchon, J., Garinet, S., \& Pasmant, E. (2016). RAS-MAPK pathway epigenetic activation in cancer: miRNAs in action. Oncotarget, 7(25), 38892-38907. http://dx.doi.org/10.18632/oncotarget.6476

Melo, S. F. S., Barauna, V. G., Neves, V. J., Fernandes, T., da Silva Lara, L., Mazzotti, D. R., \& Oliveira, E. M. (2015). Exercise training restores the cardiac microRNA-1 and -214 levels regulating $\mathrm{Ca} 2+$ handling after myocardial infarction. BMC cardiovascular disorders, 15(1), 166. http://dx.doi.org/10.1186/s12872-015-0156-4

Murray, M. J., Bell, E., Raby, K. L., Rijlaarsdam, M. A., Gillis, A. J., Looijenga, L. H., ... \& Coleman, N. (2016). A pipeline to quantify serum and cerebrospinal fluid microRNAs for diagnosis and detection of relapse in paediatric malignant germ-cell tumours. British journal of cancer, 114(2), 151-162. http://dx.doi.org/10.1038/bjc.2015.429

Naito, Y., Tanaka, Y., \& Ochiya, T. (2015). microRNAs and Hepatitis B. Adv Exp Med Biol., 888, 389-399.

Pilmore, E., \& Hamilton, K. L. (2015). The Role of MicroRNAs in the Regulation of K+ Channels in Epithelial Tissue. Frontiers in physiology, 6, 1-7.

Rabiee-Ghahfarrokhi, B., Rafiei, F., Niknafs, A. A., \& Zamani, B. (2015). Prediction of microRNA target genes using an efficient genetic algorithm-based decision tree. FEBS open bio, 5, 877-884.

Rath, S., Donovan, J., Whitney, G., Chitrakar, A., Wang, W., \& Korennykh, A. (2015). Human RNase L tunes gene expression by selectively destabilizing the microRNA-regulated transcriptome. Proceedings of the National Academy of Sciences, 112(52), 15916-15921.

Selitsky, S. R., Dinh, T. A., Toth, C. L., Kurtz, C. L., Honda, M., Struck, B. R., ... \& Sethupathy, P. (2015). Transcriptomic analysis of chronic hepatitis $\mathrm{B}$ and $\mathrm{C}$ and liver cancer reveals microRNA-mediated control of cholesterol synthesis programs. MBio, 6(6), e01500-15. http://dx.doi.org/10.1128/mBio.01500-15

Sun, D. K., Wang, J. M., Zhang, P., \& Wang, Y. Q. (2015). MicroRNA-138 regulates metastatic potential of bladder cancer through ZEB2. Cellular Physiology and Biochemistry, 37(6), 2366-2374.

Tang, H., Wu, H., Yang, Y., Zhao, J., \& Chen, J. (2015). Progress in study on the role of exosome-derived microrna in diagnosis and treatment of diseases. Zhong nan da xue xue bao. Yi xue ban=Journal of Central South University. Medical sciences, 40(11), 1270-1275. ttp://dx.doi.org/10.11817/j.issn.1672-7347.2015.11. 018

Tuna, M., Machado, A. S., \& Calin, G. A. (2016). Genetic and epigenetic alterations of microRNAs and implications for human cancers and other diseases. Genes, Chromosomes and Cancer, 55(3), 193-214. http://dx.doi.org/10.1002/gcc.22332.

Virant-Klun, I., Ståhlberg, A., Kubista, M., \& Skutella, T. (2016). MicroRNAs: from female fertility, germ cells, and stem cells to cancer in humans. Stem cells international, 2016. http://dx.doi.org/10.1155/2016/3984937

Wang, X., Chen, K., Guo, G., \& Chen, J. L. (2016). Noncoding RNAs and their functional involvement in regulation of chronic myeloid leukemia. Briefings in functional genomics, elv059.

Xiao, J., Bei, Y., Liu, J., Dimitrova-Shumkovska, J., Kuang, D., Zhou, Q., ... \& Yang, C. (2016). miR-212 downregulation contributes to the protective effect of exercise against non-alcoholic fatty liver via targeting FGF-21. Journal of cellular and molecular medicine, 20(2), 204-216. http://dx.doi.org/10.1111/jcmm.12733

Xiao, J., Wang, Q. X., \& Zhu, Y. Q. (2015). Altered expression profile of micrornas in gastric stromal tumor. Journal of Huazhong University of Science and Technology [Medical Sciences], 35(6), 842-850.

Yu, X., \& Li, Z. (2016). The role of microRNAs in the adrenocortical carcinomas. Tumor Biology, 37(2), 15151519. http://dx.doi.org/10.1007/s13277-015-4630-5

Zhang, F., Xu, Z., Wang, K., Sun, L., Liu, G., \& Han, B. (2015). microRNA and gene networks in human laryngeal cancer. Experimental and therapeutic medicine, 10(6), 2245-2252.

Zou, X., Zhong, J., Li, J., Su, Z., Chen, Y., Deng, W., ... \& Li, Z. (2016). miR-362-3p targets nemo-like kinase and functions as a tumor suppressor in renal cancer cells. Molecular medicine reports, 13(1), 994-1002. http://dx.doi.org/10.3892/mmr.2015.4632

\section{Copyrights}

Copyright for this article is retained by the author(s), with first publication rights granted to the journal.

This is an open-access article distributed under the terms and conditions of the Creative Commons Attribution license (http://creativecommons.org/licenses/by/4.0/). 